\title{
Clinical observation of Pemetrexed first-line treatment in advanced non-squamous lung cancer or non-small cell lung cancer without driver-mutations: a phase 2 , single-arm trial
}

\author{
Zhou Sha ${ }^{1 \#} \wedge$, Jian-Bo He ${ }^{2 \#}$, Qinling Jiang ${ }^{3}$, Linlin $\mathrm{Xu}^{2}$, Liyang $\mathrm{Hu}^{1}$, Zibin Liang ${ }^{1}$, Tin $\mathrm{Li}^{1}$, Zhong Lin ${ }^{1}$, \\ Qitao $\mathrm{Yu}^{2}$, Xiaofeng Pei ${ }^{1}$, Weize $\mathbf{L v}^{1}$ \\ ${ }^{1}$ Department of Thoracic Oncology, The Cancer Center of The Fifth Affiliated Hospital of Sun Yat-sen University, Zhuhai, China; ${ }^{2}$ Guangxi \\ Medical University Cancer Hospital, Nanning, China; ${ }^{3}$ Department of Oncology, Nanxishan Hospital of Guangxi Zhuang Autonomous Region, \\ Guilin, China \\ Contributions: (I) Conception and design: All authors; (II) Administrative support: All authors; (III) Provision of study materials or patients: All \\ authors; (IV) Collection and assembly of data: All authors; (V) Data analysis and interpretation: All authors; (VI) Manuscript writing: All authors; (VII) \\ Final approval of manuscript: All authors. \\ \#These authors contributed equally to this work. \\ Correspondence to: Weize Lv. Department of Thoracic Oncology, The Cancer Center of The Fifth Affiliated Hospital of Sun Yat-sen University, 52 \\ Meihua Road, Zhuhai 519000, China. Email: lvweize@163.com; Xiaofeng Pei. Department of Thoracic Oncology, The Cancer Center of The Fifth \\ Affiliated Hospital of Sun Yat-sen University, 52 Meihua Road, Zhuhai 519000, China. Email: pxf3@mail.sysu.edu.cn.
}

Background: Non-dominant population, which means patients with advanced non-squamous lung cancer or non-small cell lung cancer (NSCLC) without driver-mutations, who are excluded from clinical studies because of specific baseline conditions refractory to multiple treatments, have poor outcomes. We assessed the activity of pemetrexed first-line treatment for a non-dominant population, explore the safety and efficacy of pemetrexed therapy.

Methods: We did this two-phased, single-arm trial at two sites at the Fifth Affiliated Hospital of Sun Yat-sen University and Guangxi medical university cancer hospital. Pemetrexed $500 \mathrm{mg} / \mathrm{m}^{2}$, static drops on day 1;21 days for a cycle, each treatment for at least two cycles and up to six cycles. Efficacy was assessed every two cycles.

Results: We counted the July 21, 2018 to 2020 on May 31, first diagnosed with IIIb-IV period (American Joint Committee on Cancer eighth edition) no drive genes, non-squamous cell carcinomas, 30 patients with non-small cell lung cancer, the follow-up to July 31, 2020, median follow-up time was 12 months. Most were elderly patients with poor general conditions (96.7\% of patients had ECOG scores of 2-3) (median age 66 years). Median duration of maintenance treatment was 6 months. Median progression-free survival was 6.5 months. Median overall survival was 12 months. Patients with performance status $=0-2$ had a significantly higher median overall survival time (16 months) compared with patients with performance status $=3$ who had a median overall survival time of 7 months $(\mathrm{P}=0.001)$. Most treatment-related adverse events were grade 1 or grade 2.

Conclusions: This study is the first to investigate the survival benefit and toxicity tolerance of pemetrexed treatment in non-dominant population in the real world, providing a new therapeutic possibility for those who failed to be enrolled in clinical studies.

Keywords: Pemetrexed; non-small cell lung cancer (NSCLC); non-dominant population

Submitted Aug 21, 2020. Accepted for publication Oct 19, 2020.

doi: $10.21037 /$ atm-20-6539

View this article at: http://dx.doi.org/10.21037/atm-20-6539

^ ORCID: Zhou Sha, 0000-0002-5798-1837; Jian-Bo He, 0000-0002-7413-9414. 


\section{Introduction}

Lung cancer is the most common cancer with the highest mortality rate. Non-small cell lung cancer (NSCLC) accounts for about $80 \%$ of lung cancer. Because of its high malignancy and rapid development, $70-80 \%$ of NSCLC patients are inoperable when diagnosed, and comprehensive treatment based primarily on systemic treatment is often required. Driver gene is an important gene related to the development of lung cancer. In recent years, with the development of pharmacogenomics, in addition to common EGFR and ALK drivers have been found to be effective in the use of targeted therapeutic drugs, other driver genes have also been found successively, such as MET, HER-2, RET, KRAS, BRAF, NTRK, MEK, TROP2 overexpression, the emergence of targeted therapy has promoted the treatment effect of NSCLC, which accounts for nearly $80 \%$ of lung cancer. However, not every tumor patient is suitable for targeted therapy and chemotherapy is still an important treatment method for advanced NSCLC with the emergence of targeted drug resistance.

At present, chemotherapy is the primary first-line treatment in patients with advanced non-squamous lung cancer or NSCLC without driver-mutations. Guidelines recommend patients with no progression after 4-6 cycles of two-drug chemotherapy regimen with pemetrexed should receive maintenance therapy with pemetrexed $(1,2)$ or three-drug regimen with carboplatin + paclitaxel + bevacizumab, bevacizumab should be used until progression (3-8), or platinum-containing double-drug regimen with bevacizumab $(9,10)$. These recommendations are with the premise that the patient has a good performance status (PS) score of 0-2 or ECOG of 0-2, adequate compliance, and regular biochemical indexes. Patients who meet these criteria are the dominant population for clinical trials. However, in reality, the conditions of patients are often not as standard as the inclusion conditions of clinical research. There also existed a particular group of people who are excluded from clinical studies for their specific baseline conditions or limitations by their concomitant conditions, which we call the non-dominant population. In this group, some patients are not willing to accept an intense chemotherapy regimen due to poor compliance, weak psychological endurance ability, fear, and anxiety for chemotherapy. The others had a PS score $>2$, or cardiopulmonary, liver or kidney functions were slightly beyond the range specified in the guidelines, but their expected survival time was $>3$ months, and have a strong desire for treatment. For the non-dominant population, guidelines recommend optimal supportive care. However, we have found that it is not enough in clinical. Also, we found that low-dose and low-toxicity chemotherapy drugs can control the tumor, relieve symptoms and improve the quality of life for some patients with ovarian cancer or small-cell lung cancer in poor general conditions in our preliminary clinical work. Therefore, this clinical study uses low-dose and low-toxicity pemetrexed to control the cancer progression, reduce symptoms and improve the quality of life for the non-dominant population with advanced nonsquamous lung cancer or NSCLC without driver-mutations and excluded from the clinical trials for a more prolonged overall survival (OS) and progression-free survival (PFS).

Pemetrexed is a newly developed, antifolic acid agent. In tumor cells, the folic acid metabolism of dependency is crucial. Pemetrexed can inhibit folate-dependent metabolism in multiple targets as a tumor-suppressor. Pemetrexed can effectively inhibit various enzymes in metabolic pathways, promote the apoptosis of tumor cells, and play an anti-cancer effect. Early researches have shown pemetrexed can powerfully inhibit thymidylic acid synthase (TS) from reducing the synthesis of uracil, dihydrofolate reductase (DHFR) to block the synthesis of tetrahydrofolate and the activity of GARFT to interfere the anabolism of purine. These enzymes are involved in the synthesis of DNA and RNA in tumor cells, while pemetrexed can inhibit these key enzymes effectively, create tumor cells stop at $\mathrm{S}$ stage, and promotes their apoptosis. Clinical studies have shown that pemetrexed has significant anti-cancer activity against various tumors, including colorectal cancer, lung cancer, and pancreatic cancer. The American Society of Clinical Oncology recommends using pemetrexed combined with platinum-based chemotherapy as a first-line treatment. According to the final survival analysis of the PARAMOUNT trial, continued pemetrexed maintenance therapy improved survival in patients with advanced nonsquamous NSCLC compared with placebo (11), In recent years, there have been many cases of NSCLC treated with pemetrexed and dual-drug chemotherapy, with apparent effects and few adverse reactions, which are highly effective and low-toxicity chemotherapy drugs.

Our study aims to research pemetrexed first-line treatment in patients with advanced non-squamous lung cancer or NSCLC without driver-mutations, explore the safety and efficacy of pemetrexed therapy, and observe the changes of psychological status and biochemical indicators before and after treatment, and subsequent changes in 
the treatment, to find the optimal treatment for the nondominant population. The novel idea of this study was the introduction of pemetrexed maintenance into nondominant patients who might receive supportive treatment.

We present the following article in accordance with the TREND reporting checklist (available at http://dx.doi. org/10.21037/atm-20-6539).

\section{Methods}

\section{Study design and patients}

Our study is a multicenter, open, single arm clinical study; patients were first diagnosed with stage IIIb-IV (AJCC 8th) without driver-mutations, non-squamous cell carcinomas, and non-small cell lung cancer. All procedures performed in this study involving human participants were in accordance with the Declaration of Helsinki (as revised in 2013). The study was approved by ethics board of the fifth Affiliated Hospital of Sun Yat-sen University (2018-K19-1). Patients provided written informed consent. Radiation imaging, biochemical indicators, and survival state data were collected. All patients were followed until death or end of the study.

To be eligible for the study, patients must meet all the following inclusion criteria: (I) unresectable histology and cytology confirmed stage IIIb-IV (AJCC 8th) without driver-mutations, non-squamous cell carcinomas, and NSCLC; (II) age >18 years old, regardless of gender; (III) primary patients who have not received surgery, chemotherapy, biological therapy or radiation therapy; (IV) expected survival $\geq 3$ months; (V) the presence of lesions; (VI) ECOG score 0-3.

\section{Treatment plan}

Pemetrexed $500 \mathrm{mg} / \mathrm{m}^{2}$, static drops on day 1; 21 days for a cycle, each treatment for at least two cycles and up to six cycles. After two cycles of treatment, the subjects will be timely converted to a platinum-containing dualdrug regimen according to their physical condition and psychological acceptance of chemotherapy. After six cycles of treatment, patients in SD, PR, or CR received pemetrexed maintenance therapy until the disease progressed or intolerant. All patients were tested for objective response rate (RR), disease control rate (DCR), progression-free survival (PFS), 1- and 2-year survival rate, changes in PS score, and adverse reactions. Folic acid and vitamin B12 supplementation was included in the treatment, dexamethasone was used to prevent rashes caused by pemetrexed, routine antiemesis treatment was performed before and after chemotherapy, and G-CSF was used according to the condition. Imaging review and tumor lesion measurements were performed every two cycles. Blood routine, liver, renal function, electrocardiogram, and psychological assessment scale were examined before and after each cycle of chemotherapy, and various adverse reactions of chemotherapy were recorded.

\section{Instruments}

\section{Self-Rating Depression Scale (SDS) and Self-Rating Anxiety Scale (SAS)}

The anxiety and depression were self-measured among patients using the Zung Self-Rating Anxiety Scale and the Self-Rating Depression Scale (Z-SDS) $(12,13)$. The subjects rated each item on a four-point scale with how they had felt in the previous week, with four showing the least satisfying response. The sum of the 20 items produced an original score converted to a self-score for anxiety or depression after correcting the reverse scores of the ten items (called the "SAS"/"SDS" index). According to previous studies, Z-SDS and Z-SAS have been established as a valuable screening tool for the oncology population $(14,15)$.

All patients were asked to complete SAS and SDS assessments before and after the first pemetrexed chemotherapy cycle. For uneducated patients, nurses were trained to collect data from patients and their relatives.

\section{PS}

An important indicator of general health status is the evaluation of PS. The ECOG score is an indicator of a patient's general health status and tolerance to treatment with their physical strength. ECOG Physical Fitness rating scale scores: $0,1,2,3,4$ and 5 points.

All patients were asked to complete SAS and SDS assessments before and after the first pemetrexed chemotherapy cycle. For uneducated patients, nurses were trained to collect data from patients and their relatives.

\section{Statistical analysis}

The baseline data include demographic characteristics, baseline tumor characteristics, medical history, and vital signs. Means describe the measurement data, standard deviation, median, minimum, and maximum. For counting 


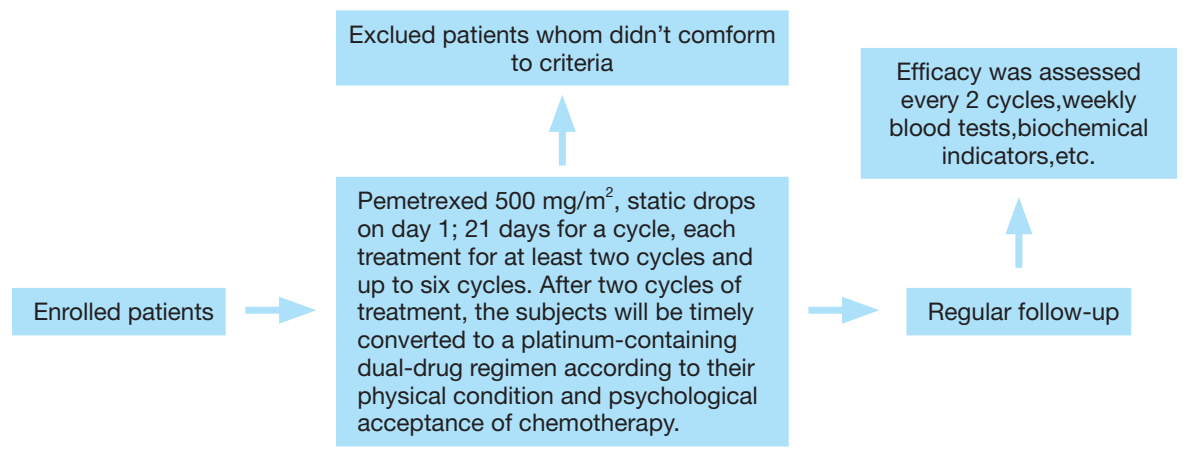

Figure 1 Trial profile. Pemetrexed $500 \mathrm{mg} / \mathrm{m}^{2}$, static drops on day 1;21 days for a cycle, each treatment for at least two cycles and up to six cycles. After two cycles of treatment, the subjects will be timely converted to a platinum-containing dual-drug regimen according to their physical condition and psychological acceptance of chemotherapy. After six cycles of treatment, patients in complete response (CR); partial response $(\mathrm{PR})$ or stable disease $(\mathrm{SD})$ received pemetrexed maintenance therapy until the disease progressed or intolerant.

data, frequency and percentage are used to describe. $T$-test or Wilcoxon rank-sum test, chi-square test, and Fisher's exact probability were used for comparison of baseline indicators between groups.

After the data were collected, Fisher's exact probability method or Chi-square test was used for statistical analysis of dichotomous data and other classified data. Kaplan-Meier curve was used to analyze the time to 2 degrees or more toxic adverse reactions. The statistical parameter was a $\mathrm{P}$ value of 0.05 .

\section{Results}

\section{Patients}

Between July 1, 2018, and May 31, 2020, 30 patients were enrolled (Figure 1). Data are presented up to July 31, 2020. Patients baseline characteristics are shown in Table 1. Most patients were women $(56.7 \%)$, with a median age of 66 years. Ten patients (33.3\%) were current or former smokers. $93.3 \%$ of the patients had stage IV disease, $46.7 \%$ of the patients had an ECOG PS of 2, and 33.3\% had an ECOG PS of 3. Most patients (29 of 30) were adenocarcinoma, 27 patients had no mutation (according to the state of gene mutation before inclusion), while three patients had mutations for unclassical EGFR mutation (20 exon insertion).

The duration of pemetrexed therapy is shown in Figure 2. The median duration of treatment was 2 months. Six patients $(20 \%)$ were treated with pemetrexed beyond 6 months. Only one patient was treated with pemetrexed beyond 1 year. At the time of analysis, no one remained on pemetrexed. Seventeen $(56.7 \%)$ patients changed regimen to two-drug chemotherapy regimen with pemetrexed.

\section{Clinical activity}

With a median follow-up of 12 months (IQR, 2-30 months), initial objective responses were all observed after two cycles of pemetrexed treatment (shown in Figure 3). Most patients had an SD after the first post-baseline assessment (16, $53.3 \%)$. Eight (26.7\%) had PD, and $2(6.67 \%)$ had a PR as the best response. Four (13.3\%) of 30 patients were not evaluable for a response from the preliminary post-baseline assessment.

At the time of the data cutoff, all patients $(90 \%)$ had disease progression (Figure $4 A$ ). The median PFS was 6.5 months (95\% CI: $1-21)$. There were 26 death eventsthe median survival was 12 months (95\% CI: 2-not reached, Figure 4B).

\section{PS and survival}

Before treatment, the baseline ECOG score was $66.7 \%$ of the patients $(n=20)$ had an ECOG PS of $0-2$, and $33.3 \%(n=10)$ had an ECOG PS of 3. After two cycles of pemetrexed treatment, the percentage hardly changed, showing that It suggests that most patients are well tolerated to pemetrexed treatment.

Patients with PS $=0-2$ had a median PFS of 7 months, and it was 5 months for patients with PS $=3$, showing a difference without statistical significance $(\mathrm{P}=0.0549)$ (Figure $5 A)$. However, in a 3-year follow-up, patients with PS $=0-2$ had a significantly higher median OS time (16 months) compared with patients with PS $=3$ who had a median OS time of 
Table 1 Baseline patient and disease characteristics by treatment period $(\mathrm{N}=30)$

\begin{tabular}{|c|c|}
\hline Characteristics & Value, n (\%) \\
\hline \multicolumn{2}{|l|}{ Sex } \\
\hline Male & $13(43.3)$ \\
\hline Female & $17(56.7)$ \\
\hline \multicolumn{2}{|l|}{ Age } \\
\hline$<65$ years & $14(46.7)$ \\
\hline$\geq 65$ years & $16(53.3)$ \\
\hline \multicolumn{2}{|l|}{ Smoking status } \\
\hline Never smoker & $20(66.7)$ \\
\hline$>0-15$ pack-years & $2(6.67)$ \\
\hline$>15$ pack-years & 8 (26.7) \\
\hline \multicolumn{2}{|l|}{ ECOG PS } \\
\hline 0 & $2(6.67)$ \\
\hline 1 & $4(13.3)$ \\
\hline 2 & $14(46.7)$ \\
\hline 3 & $10(33.3)$ \\
\hline \multicolumn{2}{|l|}{ Disease stage } \\
\hline IIIB & $2(6.67)$ \\
\hline IV & $28(93.3)$ \\
\hline \multicolumn{2}{|c|}{ Histology at first diagnosis } \\
\hline Adenocarcinoma & $29(96.7)$ \\
\hline Other & $1(3.3)$ \\
\hline \multicolumn{2}{|l|}{ Mutations } \\
\hline EGFR & $3(10.0)$ \\
\hline ALK & $0(0.0)$ \\
\hline Others & $0(0.0)$ \\
\hline Unknown & $0(0.0)$ \\
\hline Negative & $27(90.0)$ \\
\hline
\end{tabular}

ECOG, Eastern Cooperative Oncology Group score standard; PS, performance status; EGFR, epidermal growth factor receptor; ALK, anaplastic lymphoma kinase.

7 months $(\mathrm{P}=0.001)$ (Figure $5 B)$.

\section{Psychological states}

According to Z-SDS evaluation, the baseline prevalence of prechemotherapy depression in NSCLC patients was $30 \%$

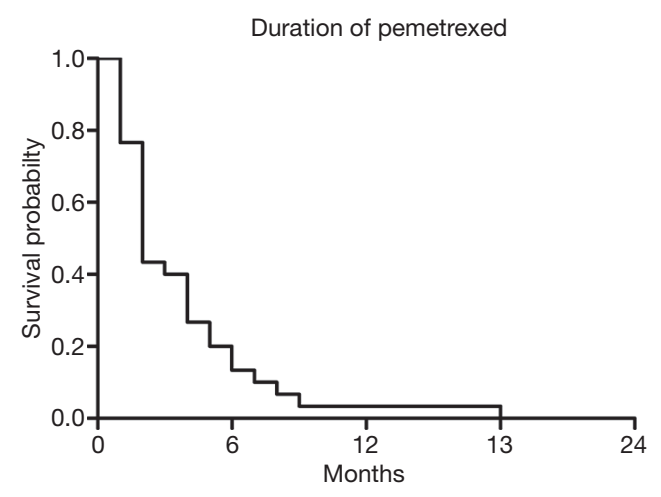

Figure 2 Duration of pemetrexed therapy, the median duration of treatment was 2 months.

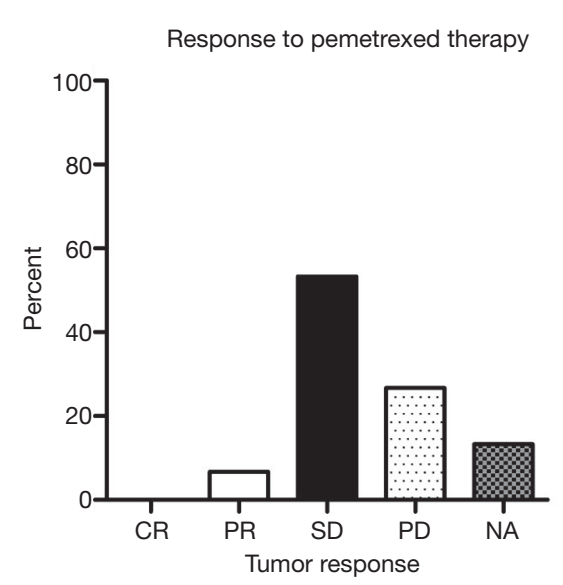

Figure 3 Tumor response after two cycles of pemetrexed treatment. CR, complete response; PR, partial response; SD, stable disease; $\mathrm{PD}$, progressive disease; NA, no evaluation

( $N=9$, mean $=49$ ), while the baseline prevalence of anxiety was $40 \%$ ( $N=12$, SDS: 47.2).

After two cycles of pemetrexed treatment, the prevalence of depression decreased to $13.3 \%(\mathrm{~N}=4$, SAS: 38.3$)$; there is no significant difference $(\mathrm{P}=0.3265)$. The prevalence of anxiety decreased to $26.7 \%(\mathrm{~N}=8$, SAS: 41$)$ among patients according to Z-SAS evaluation, $\mathrm{P}=0.8441$ (Figure 6).

\section{Safety and adverse events}

Twenty-nine patients were treated for adverse events. Of all patients, most treatment-related adverse events were grade 1 or grade 2 (Table 2), and one or more drug-related toxicities of any grade were observed in 29 patients (overall toxicity rate of $96.2 \%, 95 \%$ CI: $80.4-99.9 \%$ ). The most common 

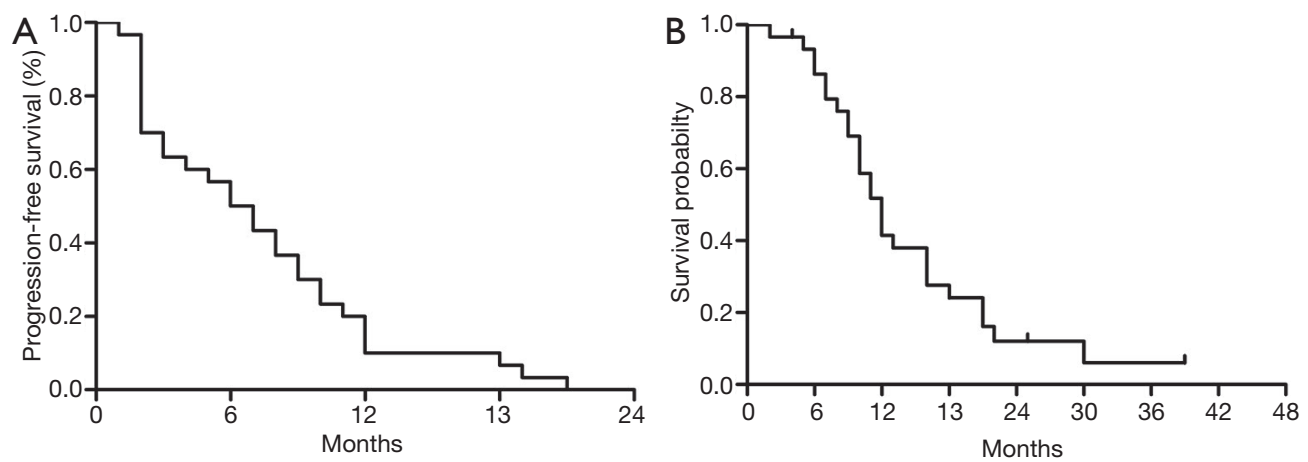

Figure 4 Survival analysis of non-dominant patients. (A) Progression-free survival curve, median progression-free survival was 6.5 months; (B) overall-survival curve, median overall survival was 12 months.
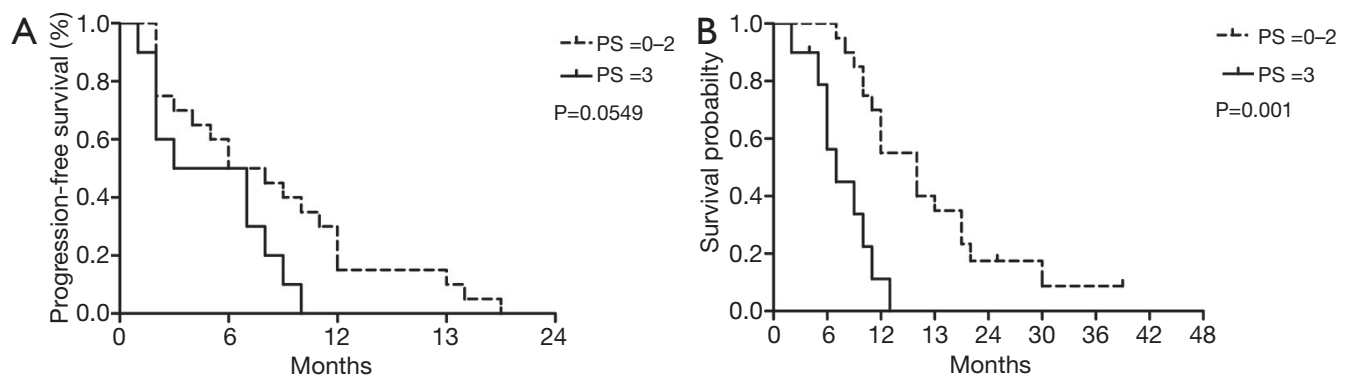

Figure 5 Survival analysis of non-dominant patients with PS 0-2 and PS =3. (A) Progression-free survival curve, log-rank level of compared factors, $\mathrm{P}=0.0549$; (B) overall-survival curve, log-rank level of compared factors, $\mathrm{P}=0.001$. PS, performance status.

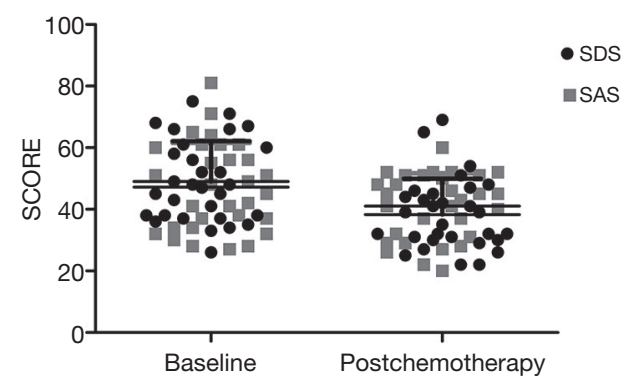

Figure 6 SAS and SDS score of patients before treatment and after two cycles of pemetrexed therapy $(\mathrm{P}>0.05)$. SAS, Self-Rating anxiety scale; SDS, Self-Rating depression scale

treatment-related adverse events were increased alanine aminotransferase, increased aspartate aminotransferase, decreased platelet count, decreased leukocyte count, and decreased neutrophil count. Patients in whom these adverse events were observed were asymptomatic, and these adverse events were resolved with dose modification. No grade 3 or worse treatment-related events were observed. No drug-related deaths were observed. The reasons for deaths during follow up on this study were predominantly disease progression (23 patients), and acute respiratory failure in one patient, and two from a suspected disease-related pulmonary embolism.

\section{Discussion}

NSCLC is the leading cause of cancer mortality globally, with about 2.2 million recent cases and 1.6 million deaths each year (16). NSCLC is the leading cause of cancer mortality globally, with about 2.2 million recent cases and 1.6 million deaths each year (17). At present, the first-line treatment for advanced non-driver gene, nonsquamous cell carcinoma, and NSCLC is chemotherapy, especially dual-drug chemotherapy with pemetrexed. With the development of precision therapy for lung cancer, clinical trials of various new drugs and new treatment regimens have become the alternative for patients with advanced lung cancer. However, in the actual world, some people are excluded from clinical studies through special 
Table 2 Adverse effects of chemotherapy in patients after two cycles of pemetrexed therapy

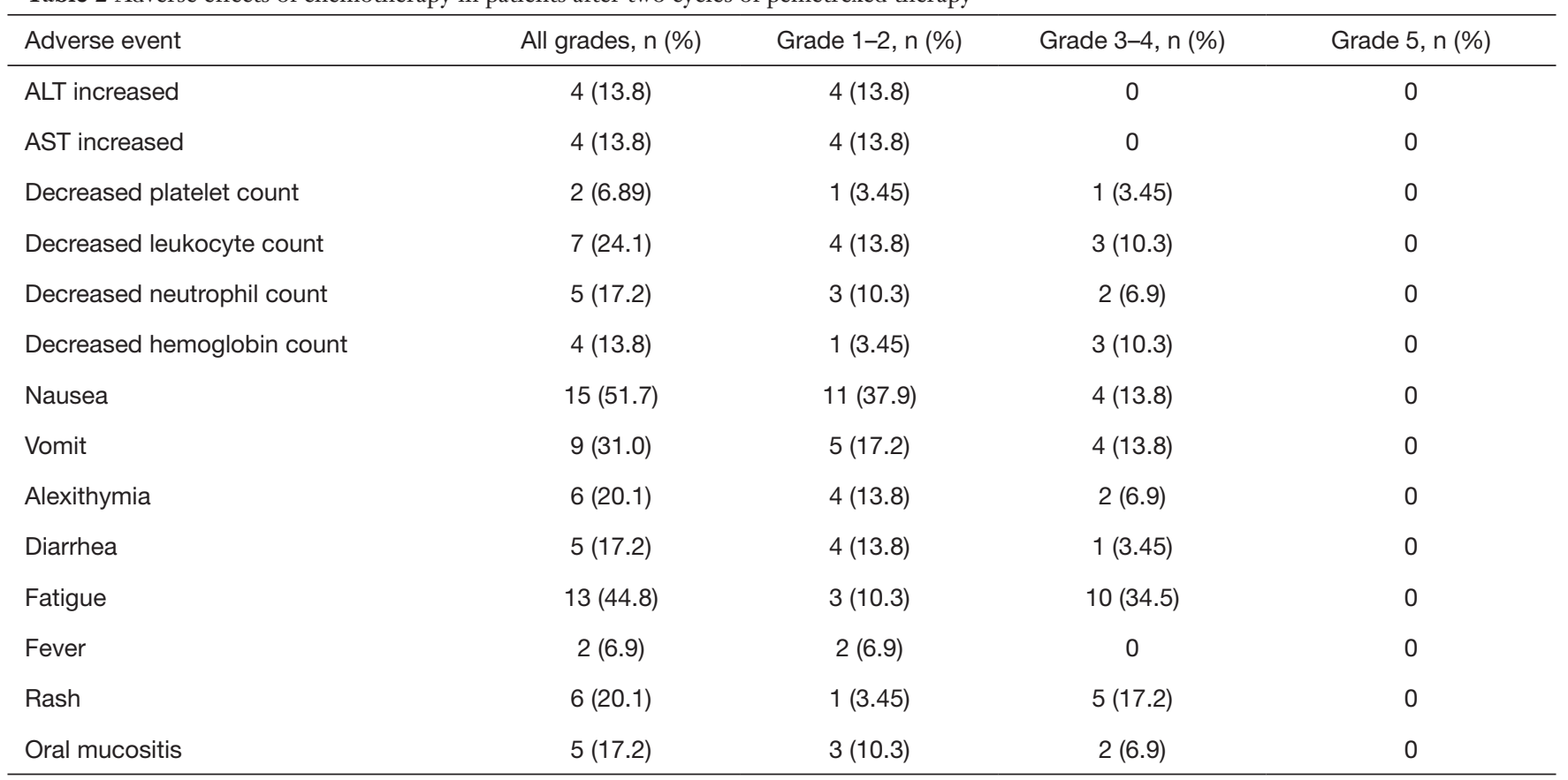

$\mathrm{ALT}$, alanine aminotransferase; AST, aspartate aminotransferase.

baseline treatment conditions, self-associated diseases, or unsatisfactory PS status, which we define as a non-dominant population. For such patients, the guidelines recommend optimal supportive care. However, for patients with an expected survival period longer than 3 months and a strong desire for treatment, the effect of palliative treatment alone on improving prognosis and prolonging survival is minimal, which urges us to explore the best treatment plan for this non-dominant group in clinical work.

According to the American Society of Clinical Oncology Clinical Practice Guidelines on Chemotherapy for stage IV, NSCLC: recommendations were for the treatment strategies that improve OS. Treatments that improve only PFS prompted scrutiny of toxicity and quality of life. In NSCLC patients with stage IV, first-line cytotoxic chemotherapy should be stopped at disease progression or after four cycles, in patients whose disease is stable but not responding to treatment. Pemetrexed is recommended as second-line therapy $(17,18)$. A randomized phase III trial of gemcitabine and pemetrexed as first-line treatments for advanced NSCLC showed pemetrexed was like Gmetrix, similar in health-related quality of life (HRQoL) and survival effects, and less toxic and more convenient (19). In 2008, Scagliotti et al. first compared the efficacy of cisplatin/pemetrexed in first-line treatment with cisplatin/gemcitabine in
NSCLC patients. Nineteen In this study, both groups had the same OS (10.3 months), but cisplatin/pemetrexed significantly prolonged survival in the non-squamous cell subgroup (adenocarcinoma at 12.6 and 10.9 months, macro histology at 10.4 and 6.7 months) (20). Pemetrexed can also be used in maintenance therapy. Phase III trial that compared pemetrexed with placebo as maintenance therapy after four cycles of platinum-based doublet chemotherapy (docetaxel, gemcitabine, or paclitaxel) found longer PFS (4.3 months with pemetrexed versus 2.6 months with placebo; $\mathrm{HR}=0.50 ; 95 \% \mathrm{CI}: 0.42-0.61$ ) and $\mathrm{OS}$ (13.4 months with pemetrexed versus 10.6 months with placebo; $\mathrm{HR}=0.79 ; 95 \% \mathrm{CI}: 0.65-0.95)(21)$. The PARAMOUNT study compared the efficacy of pemetrexed maintenance therapy and placebo in stage IV NSCLC patients treated with cisplatin and pemetrexed first-line dual-drug chemotherapy. The study found pemetrexed had longer PFS than placebo (3.9 vs. 2.6 months for placebo; $\mathrm{HR}=0.64 ; 95 \%$ confidence interval, 0.51-0.81). The level 3-5 toxicological side effects of the two groups were similar, except for hematological side effects. Quality of life was similar in both groups (22). The efficacy of pemetrexed as a second-line treatment of advanced NSCLC was validated in a phase III randomized clinical trial that compared pemetrexed with docetaxel. In this study, Hanna et al. 
proved the non-inferiority of Peimetrel and Docetaxel in the second-line treatment of NSCLC. The median PFS was 2.9 months and the median survival time was 8.3 and 7.9 months ( $\mathrm{P}$ values were not statistically significant) (23). A subset analysis of this study, conducted in elderly patients, showed similar results (24).

The toxicity of pemetrexed is mild. Scagliotti et al.'s phase III study (20) showed that the critical blood level 3 or 4 drug-related toxicity of cisplatin/pemetrexed was significantly lower than cisplatin/gemcitabine (neutropenia: $15 \%$ vs. $27 \%$, anemia: $6 \%$ vs. $10 \%$, thrombocytopenia: $4 \%$ vs. $13 \% ; \mathrm{P}<0.001)$. Compared with cisplatin/pemetrexed, drug-related febrile neutropenia of grade 3 or 4 ( $1 \%$ and $4 \%$, respectively; $\mathrm{P}<0.002$ ) and hair loss (all levels, $12 \%$, and $21 \%$, respectively; $\mathrm{P}<0.001$ ) was significantly lower. The major side effects of pemetrexed are myelosuppression manifested as neutropenia and thrombocytopenia, which are dose-limiting toxicities of the drug and are associated with elevated plasma homocysteine pretreatment levels (a marker of folic acid deficiency). Transaminase and bilirubin were elevated in $10-15 \%$ of patients; however, this increase is usually transient and asymptomatic (25). Pemetrexed is an agent with low emesis risk. Pemetrexed toxicity in elderly patients is controllable, reversible, and has an adequate safety profile. There is little difference in hematological toxicity between different age groups, and most of the hematological toxicity rates are low (26). Pemetrexed has also been studied for first-line treatment of special subpopulations. For elderly patients, pemetrexed alone showed a substantial antitumor activity (response rate $=25 \%)(27)$.

Therefore, in this multicenter, open, one-arm, prospective clinical study, we explored the efficacy of low-dose, lowtoxicity pemetrexed drug to control disease progression, reduce symptoms, improve quality of life, and further extend survival time for a non-dominant advanced non-driver, nonsquamous and NSCLC "excluded from clinical studies". We counted the fifth affiliated hospital of Sun Yat-sen University and Guangxi medical university cancer hospital, the July 21, 2018 to 2020 on May 31, first diagnosed with IIIb-IV period (AJCC eighth edition) no drive genes, non-squamous cell carcinomas, 30 patients with NSCLC, the follow-up to July 31, 2020, median follow-up time was 12 months. Most of them were elderly patients with poor general conditions (96.7\% of patients had ECOG scores of 2-3) (median age 66 years). The results showed that most patients could tolerate pemetrexed treatment, and the median duration of maintenance treatment was 6 months. Seventeen (56.7\%) patients changed regimen to two-drug chemotherapy regimen with pemetrexed. The median PFS was 6.5 months (95\% CI: 1-21). The median OS was 12 months. There was no statistical difference in the influence of PS state on PFS, while patients with $\mathrm{PS}=0-2$ had a significantly higher median OS time (16 months) compared with patients with PS $=3$ who had a median OS time of 7 months $(\mathrm{P}=0.001)$. In this study, toxicity was acceptable, of all patients, most treatment-related adverse events were grade 1 , or grade 2, most common treatment-related adverse events were increased alanine aminotransferase, increased aspartate aminotransferase, decreased platelet count, decreased leukocyte count and decreased neutrophil count. No grade 3 or worse treatmentrelated events were observed. No drug-related deaths were observed.

There is increasing evidence that depression and anxiety are associated with reduced treatment compliance and poor survival in cancer patients $(28,29)$. In several prospective studies of lung cancer patients (30-32), one study found a statistically significant relationship between a higher depression score and an increased risk of death from lung cancer (30). However, after palliative care, Temel et al. have shown that improved quality of life and increased levels of depression are the reasons for survival benefits in NSCLC patients. Our study found that pemetrexed treatment can improve the psychological state of patients. After the first two cycles of pemetrexed treatment, the psychological state of the patients showed changes. Although there was no statistical difference in the degree of anxiety and depression of patients before and after treatment, the psychological resistance to chemotherapy improved. According to a previous study, the decrease of patients' resistance to chemotherapy and the change of psychological state could improve the quality of life and treatment compliance of patients (33).

To the best of our knowledge, our study gives the first direct evidence for pemetrexed first-line treatment in patients with advanced non-squamous lung cancer or NSCLC without driver-mutations, explores the safety and efficacy of pemetrexed therapy. The key strength of this study was the introduction of pemetrexed maintenance into those patients who might receive supportive treatment. With pemetrexed monotherapy, we found the tumor was controlled in a subset of patients and that a considerable proportion of patients were subsequently converted to pemetrexed dual therapy with acceptable toxicity. The psychological state of the patients improved after two cycles of pemetrexed treatment, the resistance to chemotherapy 
was reduced, and the patients were more likely to receive follow-up treatment. One limitation of this study was the lack of a randomized control group because the patient sample size was insufficient.

In conclusion, our study shows that for non-dominant patients with advanced non-squamous lung cancer or NSCLC without driver-mutations, pemetrexed as a firstline treatment has clinically significant outcomes on OS and PFS improvements. Further, our study shows this treatment also improves the physiological and psychological status of these patients too. We recommend that non-dominant groups receive 2 to 6 cycles of pemetrexed chemotherapy. After 6 cycles of treatment, patients with stable, partial or complete remission may receive further maintenance therapy until the disease progresses or becomes intolerant. These findings will provide a new direction for further exploration of the optimal treatment over these populations and bring a new ray of light to the population that cannot be benefiting from clinical trials.

\section{Acknowledgments}

Funding: This work was funded by the Zhuhai Science and Technology Planning Project (ZH2202200004HJL) from The Fifth Affiliated Hospital of Sun Yat-sen University.

\section{Footnote}

Reporting Checklist: The authors have completed the TREND reporting checklist. Available at http://dx.doi. org/10.21037/atm-20-6539

Data Sharing Statement: Available at http://dx.doi. org/10.21037/atm-20-6539

Conflicts of Interest: All authors have completed the ICMJE uniform disclosure form (available at http://dx.doi. org/10.21037/atm-20-6539). The authors have no conflicts of interest to declare.

Ethical Statement: The authors are accountable for all aspects of the work in ensuring that questions related to the accuracy or integrity of any part of the work are appropriately investigated and resolved. All procedures performed in this study involving human participants were in accordance with the Declaration of Helsinki (as revised in 2013). The study was approved by ethics board of the fifth Affiliated Hospital of Sun Yat-sen University (2018-K19-1).
Patients provided written informed consent.

Open Access Statement: This is an Open Access article distributed in accordance with the Creative Commons Attribution-NonCommercial-NoDerivs 4.0 International License (CC BY-NC-ND 4.0), which permits the noncommercial replication and distribution of the article with the strict proviso that no changes or edits are made and the original work is properly cited (including links to both the formal publication through the relevant DOI and the license). See: https://creativecommons.org/licenses/by-nc-nd/4.0/.

\section{References}

1. Aberle DR, Berg CD, Black WC, et al. The National Lung Screening Trial: overview and study design. Radiology 2011;258:243-53.

2. Aberle DR, Adams AM, Berg CD, et al. Reduced lungcancer mortality with low-dose computed tomographic screening. N Engl J Med 2011;365:395-409.

3. Baxevanos P, Mountzios G. Novel chemotherapy regimens for advanced lung cancer: have we reached a plateau? Ann Transl Med 2018;6:139.

4. Zhan Q, Miao F, Huang R, et al. Efficacy and safety of bevacizumab combined with chemotherapy in symptomatic brain metastases from lung adenocarcinoma: a retrospective analysis. J Thorac Dis 2019;11:4725-34.

5. Carney DN. Lung cancer--time to move on from chemotherapy. N Engl J Med 2002;346:126-8.

6. Chen L, Kim JS, San Antonio B, et al. Safety outcomes in advanced non-small-cell lung cancer patients treated with first-line platinum-based regimens in the United States. J Thorac Dis 2019;11:4474-83.

7. Riedel RF, Andrews C, Garst J, et al. A phase II trial of carboplatin/vinorelbine with pegfilgrastim support for the treatment of patients with advanced non-small cell lung cancer. J Thorac Oncol 2007;2:520-5.

8. Huang Y, Wang Y, Hu D, et al. The influence of pemetrexed-based continuous maintenance therapy on survival of locally advanced and metastatic lung adenocarcinoma. Ann Transl Med 2019;7:524.

9. Paz-Ares L, de Marinis F, Dediu M, et al. Maintenance therapy with pemetrexed plus best supportive care versus placebo plus best supportive care after induction therapy with pemetrexed plus cisplatin for advanced nonsquamous non-small-cell lung cancer (PARAMOUNT): a double-blind, phase 3 , randomised controlled trial. Lancet Oncol 2012;13:247-55. 
10. Sandler A, Gray R, Perry MC, et al. Paclitaxelcarboplatin alone or with bevacizumab for non-small-cell lung cancer. N Engl J Med. 2006;355:2542-50.

11. Paz-Ares LG, de Marinis F, Dediu M, et al. PARAMOUNT: Final overall survival results of the phase III study of maintenance pemetrexed versus placebo immediately after induction treatment with pemetrexed plus cisplatin for advanced nonsquamous non-small-cell lung cancer. J Clin Oncol 2013;31:2895-902.

12. Zung WW. Depression in the normal aged. Psychosomatics 1967;8:287-92.

13. Zung WW. Factors influencing the self-rating depression scale. Arch Gen Psychiatry 1967;16:543-7.

14. Dugan W, McDonald MV, Passik SD, et al. Use of the Zung Self-Rating Depression Scale in cancer patients: feasibility as a screening tool. Psychooncology 1998;7:483-93.

15. Passik SD, Lundberg JC, Rosenfeld B, et al. Factor analysis of the Zung Self-Rating Depression Scale in a large ambulatory oncology sample. Psychosomatics 2000;41:121-7.

16. Azzoli CG, Baker S, Jr., Temin S, et al. American Society of Clinical Oncology Clinical Practice Guideline update on chemotherapy for stage IV non-small-cell lung cancer. J Clin Oncol 2009;27:6251-66.

17. Azzoli CG, Temin S, Aliff T, et al. 2011 Focused Update of 2009 American Society of Clinical Oncology Clinical Practice Guideline Update on Chemotherapy for Stage IV Non-Small-Cell Lung Cancer. J Clin Oncol 2011;29:3825-31.

18. Azzoli CG, Temin S, Giaccone G. 2011 Focused Update of 2009 American Society of Clinical Oncology Clinical Practice Guideline Update on Chemotherapy for Stage IV Non-Small-Cell Lung Cancer. J Oncol Pract 2012;8:63-6.

19. Grønberg BH, Bremnes RM, Fløtten O, et al. Phase III study by the Norwegian lung cancer study group: pemetrexed plus carboplatin compared with gemcitabine plus carboplatin as first-line chemotherapy in advanced non-small-cell lung cancer. J Clin Oncol 2009;27:3217-24.

20. Scagliotti GV, Parikh P, von Pawel J, et al. Phase III study comparing cisplatin plus gemcitabine with cisplatin plus pemetrexed in chemotherapy-naive patients with advanced-stage non-small-cell lung cancer. J Clin Oncol 2008;26:3543-51.

21. Ciuleanu T, Brodowicz T, Zielinski C, et al. Maintenance pemetrexed plus best supportive care versus placebo plus best supportive care for non-small-cell lung cancer: a randomised, double-blind, phase 3 study. Lancet 2009;374:1432-40.

22. Paz-Ares LG, Gomez-Roca C, Delord JP, et al. Phase I pharmacokinetic and pharmacodynamic dose-escalation study of RG7160 (GA201), the first glycoengineered monoclonal antibody against the epidermal growth factor receptor, in patients with advanced solid tumors. J Clin Oncol 2011;29:3783-90.

23. Hanna N, Shepherd FA, Fossella FV, et al. Randomized phase III trial of pemetrexed versus docetaxel in patients with non-small-cell lung cancer previously treated with chemotherapy. J Clin Oncol 2004;22:1589-97.

24. Weiss GJ, Langer C, Rosell R, et al. Elderly patients benefit from second-line cytotoxic chemotherapy: a subset analysis of a randomized phase III trial of pemetrexed compared with docetaxel in patients with previously treated advanced non-small-cell lung cancer. J Clin Oncol 2006;24:4405-11.

25. Niyikiza C, Baker SD, Seitz DE, et al. Homocysteine and methylmalonic acid: markers to predict and avoid toxicity from pemetrexed therapy. Mol Cancer Ther 2002;1:545-52.

26. Kim YS, Sun JM, Ahn JS, et al. The optimal duration of vitamin supplementation prior to the first dose of pemetrexed in patients with non-small-cell lung cancer. Lung Cancer 2013;81:231-5.

27. Gervais R, Robinet G, Clément-Duchêne C, et al. Pemetrexed and carboplatin, an active option in first-line treatment of elderly patients with advanced non-small cell lung cancer (NSCLC): a phase II trial. Lung Cancer 2013;80:185-90.

28. Irwin MR. Depression and insomnia in cancer: prevalence, risk factors, and effects on cancer outcomes. Curr Psychiatry Rep 2013;15:404.

29. Mitchell AJ, Chan M, Bhatti H, et al. Prevalence of depression, anxiety, and adjustment disorder in oncological, haematological, and palliative-care settings: a meta-analysis of 94 interview-based studies. Lancet Oncol 2011;12:160-74.

30. Buccheri G. Depressive reactions to lung cancer are common and often followed by a poor outcome. Eur Respir J 1998;11:173-8.

31. Faller H, Bülzebruck H, Drings P, et al. Coping, distress, and survival among patients with lung cancer. Arch Gen Psychiatry 1999;56:756-62.

32. Faller H, Schmidt M. Prognostic value of depressive 
coping and depression in survival of lung cancer patients. Psychooncology 2004;13:359-63.

33. Chen J, Li W, Cui L, et al. Chemotherapeutic Response and Prognosis among Lung Cancer Patients with and

Cite this article as: Sha Z, He JB, Jiang Q, Xu L, Hu L, Liang Z, Li T, Lin Z, Yu Q, Pei X, Lv W. Clinical observation of pemetrexed first-line treatment in advanced non-squamous lung cancer or nonsmall cell lung cancer without driver-mutations: a phase 2, singlearm trial. Ann Transl Med 2020;8(20):1315. doi: 10.21037/atm-206539 without Depression. J Cancer 2015;6:1121-9.

(English Language Editor: J. Chapnick) 